\title{
Research on the Enterprise Resource Management System and Auxiliary Performance Evaluation Mechanism based on Oracle Database Optimization and Security Enhancement
}

\author{
Lei Liu ${ }^{1}$, Hua Yang ${ }^{1}$ and Alian Wesmmian ${ }^{2}$ \\ ${ }^{1}$ College of Business Administration, Hunan University, China \\ ${ }^{2}$ College of Business Administration, Imperial College London, UK
}

\begin{abstract}
In this research, we propose the novel enterprise resource management system and the auxiliary performance evaluation mechanism based on Oracle database optimization and security enhancement. Database optimization is a systems engineering covers a very wide, when optimize the database should be applications, I/O subsystem and the operating system as the main object of optimization. From the database of system structure, software structure, schema objects as well as the specific business and technical implementation, carries on the overall consideration. We firstly introduce the database optimization techniques with its application on the large-scale Oracle structure. Parallel execution makes the certain function of the database by multiple server process to be processed simultaneously. These capabilities include create indexes, loading data and restore the database, etc. Using parallel mechanism in the process of database query will greatly improve the query speed, reduce the waiting time. Later, we analyze the enterprise resource management system and evaluation model, in practice, the role of performance indicators with a baton that kind of performance indicators, means something important, people will efforts in the direction of the indicators to measure and not measure what people will ignore anything. Combining the database technique, we optimize the traditional ERP system. The experiment result shows that our method enhances the robustness and feasibility of the traditional approaches.
\end{abstract}

Keywords: Enterprise Resource Management; Performance Evaluation; Oracle Database; Optimization and Security Enhancement; System Structure; Database Applications

\section{Introduction}

From the point of view of modern information system model and information system is inseparable. Early information system is a kind of simple data processing system, and today's information systems have been developed to set data processing, transaction management and decision support for the integration of new ERP system, ERP system based on the model, for the purpose of information management and auxiliary decisionmaking, therefore, model in the ERP system today has an irreplaceable position. ERP is to achieve leapfrog development of enterprises, promote the industrial society to information society the important means of implementing the ERP is not an economic problem, but is related to national survival and the development of the key problems during the period of social transformation, the development of enterprise information and the ERP will determine countries and regions in the information age the status and the future development of the fate of the enterprise is the main part of the ERP application, the enterprise information construction is the basis for the development of ERP, ERP is the key factor to the enterprise information. Enterprise as the basic unit of the economy, the application level of information is the key of national information construction, but also improves the core competitiveness, in line with international standards [1-3]. 
Enterprises implement computer management development to a certain degree there must be an integrated system to manage enterprise's each link, to improve efficiency of enterprises, so as to achieve aim of improve enterprise competitiveness. The outcomes of implementation of ERP is obvious, combination of ERP and e-commerce is business against other companies have a powerful weapon. However, in the process of implementing ERP and should not be underestimated price of to make enterprise really achieve a perfect result, the implementation of ERP enterprise have to strive. The two core methods of modern enterprise competitiveness are implementation of ERP and ecommerce. Electronic commerce and ERP is development direction of enterprise management information system. Companies want to be in a better position to in fierce international competition, has a good management system is important.

Oracle database is the $\mathrm{C} / \mathrm{S}$ or $\mathrm{B} / \mathrm{S}$ architecture using one of the most database, it completes the data management ability, the relationship between complete products and the distributed processing ability to receive the favor of many enterprises. Companies use the management information systems are often more than one, so there will be the two mainstream database are used at the same time, when companies want to put the data in different systems can call each other, and do not affect when alone need to develop database cross-platform solutions. In the figure one, we show the inner organization and structure of the oracle database [4].

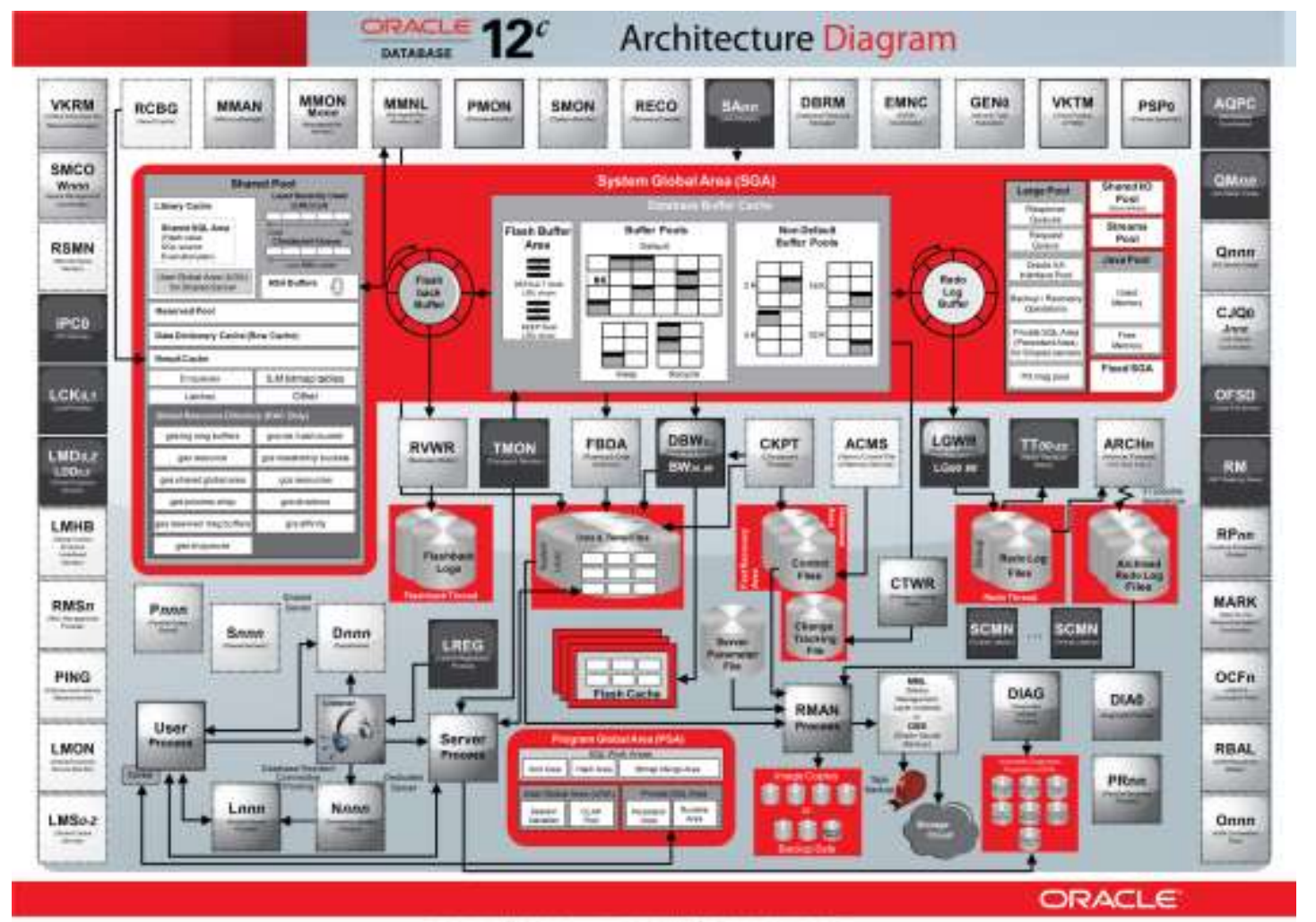

\section{Figure 1. The Inner Organization and Structure of the Oracle Database}

In terms of the Oracle database, it is powerful and the excellent performance of relational database system, one of the both in database management scale, multi-type data support, or the data parallelism of the operation or safety and stability, Oracle all look good. However, the Oracle database is also suffering from the security related challenges as the follows. (1) Safety features are mature enough. The operating system should have characteristic, but in the standard safety facilities of the database server was not there. As a result of these databases are ports can be address. The core of the operating system 
security mechanism is not applied to the database directly with the network connection. Due to the system administrator account can't be renamed, if there is no password blockade available or has been configured as the intruder to a powerful dictionary logins assault on the database server, eventually to crack the password. (2) The back door of the operating system. The characteristics of database system parameters while convenient the manager didn't, but also left a back door for database server host operating system. The Oracle database system has many useful features, can be used for direct access to operating system comes with file system. (3) Database password management issues. If the security problems exist, many of these systems passwords can let the invaders for full access to the database which will be harmful [5-7].

Currently, many of the CRM or ERP systems are using the service provided from Oracle. The following figure two demonstrates flowchart of related services. Enterprise information refers to the use of modern electronic information technology means, through the ability to improve their development and the utilization of information resources, promoting economic development, social progress and the people's own lifestyle change process. The process of enterprise information is the introduction of advanced management thought and management process. ERP software in the enterprise information system is not only the management of advanced means of order and, in fact also reflected the contemporary management thought and management idea is the crystallization of management experience of many enterprises. Therefore, the robustness and efficiency of the database is crucial for the successful system [8-11].

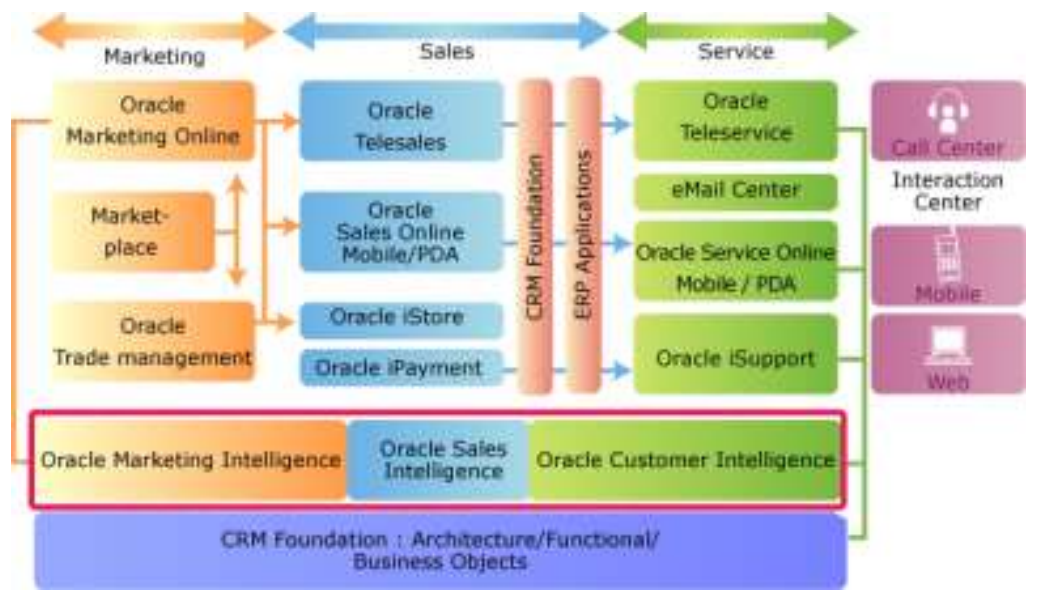

Figure 2. The Oracle Database Services for CRM and ERP Systems

To enhance the performance and safety of the traditional Oracle database based system and optimize the enterprise resource management and performance evaluation paradigm, in this research paper, we analyze the enterprise resource management system and related auxiliary performance evaluation mechanism based on the Oracle database optimization and security enhancement. Many database presents the distributed state, and most of the existing algorithm restricted by the memory and processing time as the practice is not practical, and developed a distributed algorithm is a kind of good strategy. Association rules with only raw data said that because of the low approval ratings, hard to express common data correlation. Establishing the project hierarchy, that is, mining multi-level association rules has more practical value. In the following sections we will start from the optimization of traditional database to propose our enhanced system and model step by step with in-depth discussion. 


\section{The Database Optimization Techniques}

\subsection{The Characteristics of the Large-scale Database}

Distributed database system refers to the geographical distribution and logic used in the database system, mobile database system is the inheritance and development of distributed database system. The database can be divided into the operational database and the analytical database. Operational database driven by application, geared to the needs of the specific and general transaction processing, usually need to be done in a single record level frequent insert, delete and update operation, the time response of the more demanding. When the database structure change, it will produce a lot of influence, the influence is mainly manifested in the following respects. (1) The application of the abnormal operation. Application is to access the data in the database directly, so the structure of database changes directly affect application can run normally. The advantages of this kind of direct access to the access speed are the fast; Drawback is that when the database structure because the operations such as add, delete and change, the application will appear abnormal operation. Even if the database set through unified planning has been ideal the enterprises can also according to specific need of standard local changes to database structure adjustment. (2) Increase data redundancy and complexity. In process of enterprise information development because of reason or technical knowledge database programming lack integrity, prospective, many database structure was established for different purposes in different stages, there are a lot of repeat these data to each other and brings the data redundancy. But the resulting large spelling are of the same name, different name synonymous, synonymous with the same name but different data structure problem, these problems increases complexity of data management, increased the burden of database.

Logic structure of a relational database is a collection of tables in the database, the table is composed of lines, line is a long a binary field collection. The length of the field associated with the type of field. The physical structure of the database including file group, the data table rows is storing a data file. A data file can define multiple databases as database can span multiple data files. The complete management data set is defined as the formula one [12-13].

$$
M=\sum_{i=1}^{n} I_{i}, \quad I_{i} \cap I_{j}=\varnothing
$$

Database design is for a given application environment, to construct the optimal database schema, to establish database and application system, make it can store data effectively, meet all kinds of information requirements and processing requirements of users. Large database design is a very complicated project both in the hardware, it also including the interface of the software and technology and management. Database is a collection of data, is only part of the database application system, so the database design must be combined with the application system design, in the process of the whole design to the data-driven design method based on the structure design and the function driven design method based on behavior design closely together. The density of the database size could be defined as the follows.

$$
V=i^{2} / \operatorname{Nmin}\left(M_{\text {row }}-1, M_{\text {col }}-1\right)
$$

Stored procedure is independent of the database objects outside of the table, can call by the customer, that can also call a stored procedure or from another triggers. Its parameters can be passed or returned, its error code can be tested. However, there are some of the applications, involving many server-side database processing activities, and the interaction with the user less activity, in this case, it will the processing activities in the form of stored procedures of the database on the database server, you can greatly reduce the traffic on the net, to improve application performance. Because the same physical data storage object represents meaning is the same, so they will be mapped to the same 
properties as registered in the tree node. The virtual machine will be this kind of situation report submitted to the database administrator. It is decided by the administrator for a particular purpose or its status quo to clear which reduce the redundancy in the database. The figure three shows the procedures [14-16].

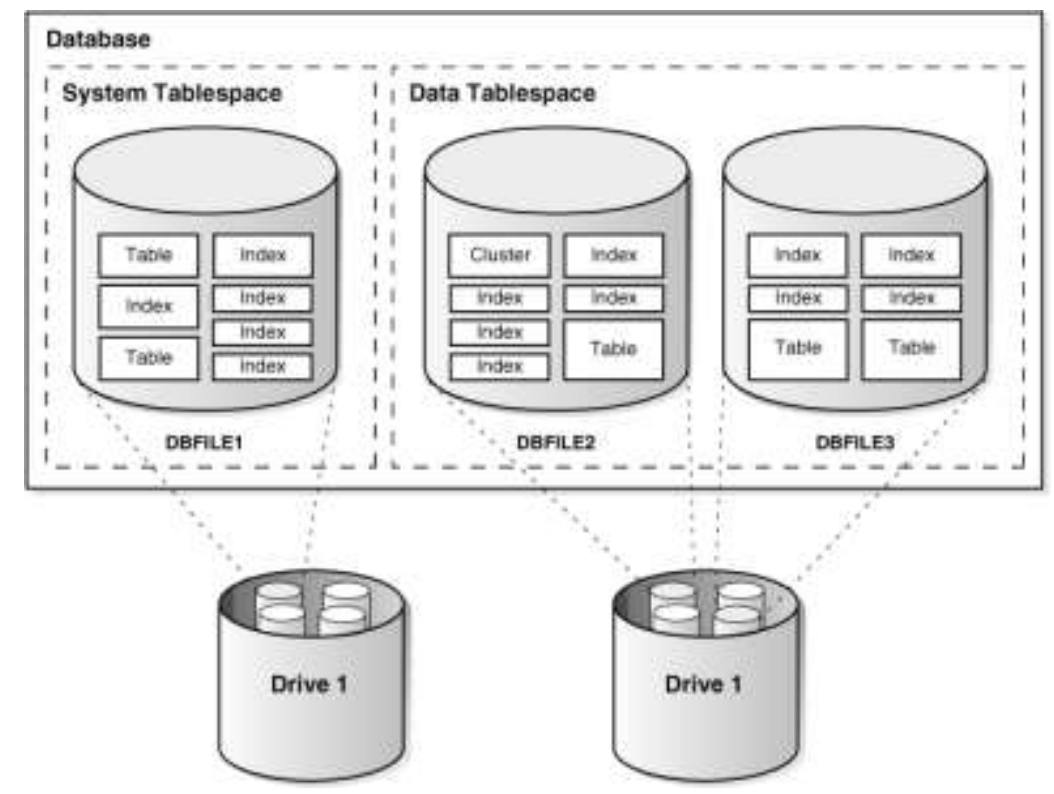

Figure 3. The Characteristics of the Large-scale Database and the Data Flow Demo

\subsection{The Database Optimization Approaches and Methods}

In the distributed database system, query optimization, including the two contents. Query optimization strategy optimization and local processing, local processing and query strategy optimization and optimization, and query strategy optimization is especially important. Even a centralized database, query execution also has a lot of ways, and in the distributed database, the same query more execution strategy. Query execution strategy as the system resource cost and response time is not the same, so query strategy of optimization is an important content of query optimization. Index is a common and important in database objects, and optimize the important method is to establish index, query table in a relational database system to establish the appropriate index, can avoid table scans and reduce I/O overhead caused by the query, improve the speed of the data query database, improve database performance. But creating an index will increase the time and space of system overhead. Therefore, create indexes must be closely combined with query requirements, and the implementation of system application can achieve the purpose of optimizing the query. Theoretically, we could optimize the designed database following the listed techniques and standards [17-18].

- $\quad$ Relationship between standardization. Relationship normalization is according to the unified standard optimize the relationship, in order to improve quality of relationship, to construct an efficient database application system to lay foundation. To construct a good database schema, must satisfy the relationship model between the properties of some inner semantic condition, and the connection can be divided into several levels of relationship of different requirements, that is relationship between standardization. The following formula demonstrates the relationship between standardization.

$$
1 N F \rightarrow 2 N F \rightarrow 3 N F \rightarrow B C N F \rightarrow 4 N F
$$

- $\quad$ Named after the specification. All the library name, table name, domain name must follow a unified naming rules, and make necessary instructions to facilitate design, 
maintenance, query. A variety of objects in the database name and the background program code should be in the form of case sensitive.

- $\quad$ The design of the primary key. It is necessary to the primary key. In practice, as often choose the smallest key group cooperation as the main key, so the primary key often is suitable for clustered index as a table. The necessity of judge index final standard is whether these indexes help in the working efficiency of the database.

- $\quad$ The design of the physical storage and the environment. At the design stage, to the physical storage of the database, the environment, the network environment to make the necessary design, make system can adapt to more in the future user concurrency and relatively large amount of data. To consider is the size of the log file, in writing when the database transaction $\log$ when a log file write full turn to another log file and the log file switch will cause the operating system overhead.

- $\quad$ The system design. System design phase should be some business logic on database programming implementation. Database programming, including the database stored procedures, triggers and functions. The benefits of database programming is used to implement the business logic is to reduce network traffic and can make full use of the database precompiled and cache function [19-20].

As an optimization example, we show sample in the figure 4. We should simplify or avoid to repeats sort data in large table if we can use index in proper order automatically generated output, the optimizer can avoid this kind of order.

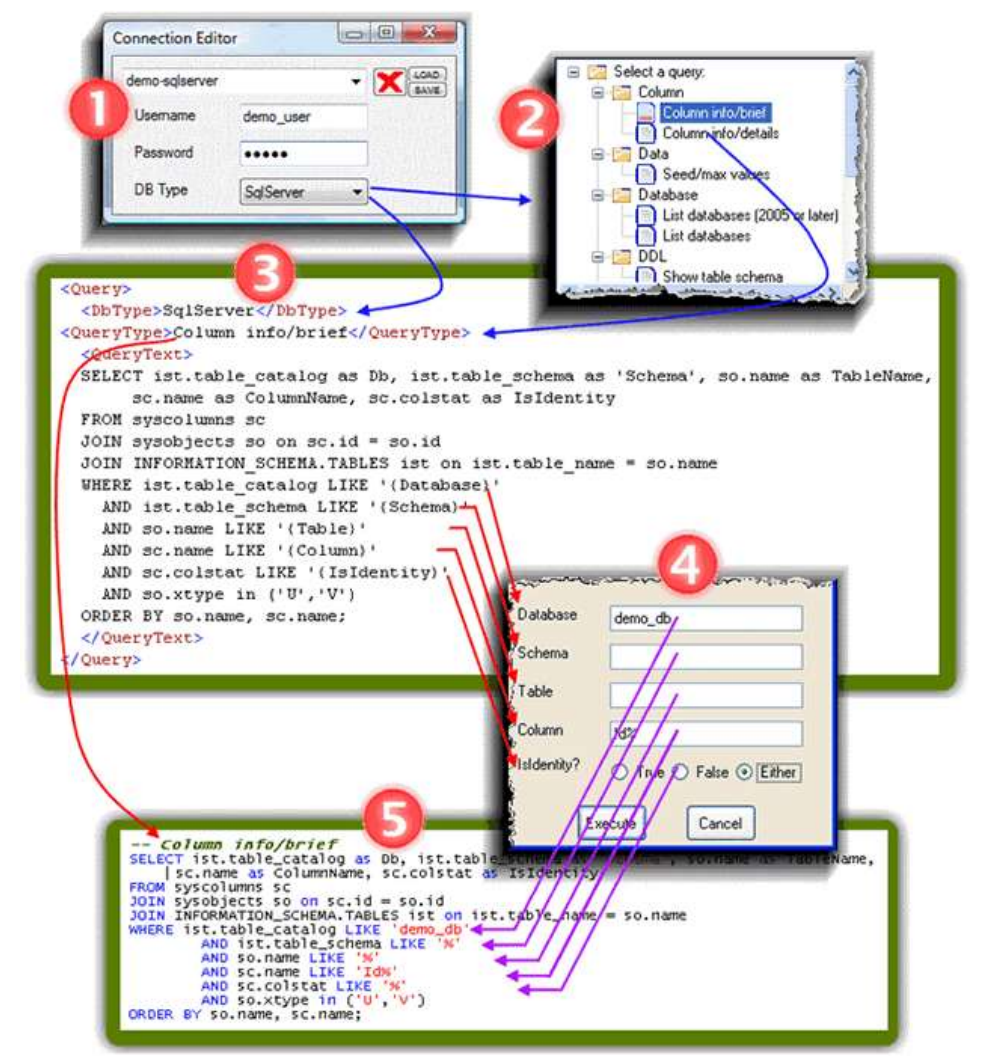

Figure 4. The Demonstration of the Database Optimization Technology 


\section{The Characteristics and Safety of the Oracle Database}

\subsection{The General Features of the Oracle Database}

Oracle performance management can be divided into two types, the active and the passive performance management. Active performance management involves the specific system implementation of early design and development, including hardware selection, performance and capacity planning, mass storage system, the choice of the I/O subsystem configuration and optimization, and how to custom of the different components, in order to meet the Oracle database and complex requirements of application system. Passive performance management involves the existing environment of the different components of the performance evaluation, troubleshooting and optimization of Oracle environment. The author puts forward how to passive performance tuning a method, in order to provide the necessary guidance for Oracle performance optimization, avoid only through trial and error method to optimize performance and improve the efficiency of the Oracle database performance management.

In daily maintenance work, if the database in the bad block, check it on the warning of a DBA can log files related to the bad block information, the DBA should first check to see if a hardware failure or operating system failure cause bad block, if not, then a database object to happen bad block and the corresponding treatment measures. In practice, there are a large number of user permissions is the same, if the database administrator for each user in every time after creating the user authorization, respectively it is a very troublesome thing, but if we have same permissions the user focused on the role of management, will be more convenient. For a role for rights management is equivalent to the role of all members, can have the same rights as a user build a character, and then for the role granted the appropriate permissions.

Just for the benefits of using roles are the system administrator permissions type, then the different authority to different roles, and don't need to care about what are the specific user, and as members of the role changes, such as adding members or delete members, system administrator need not do anything about operation of the permissions. Oracle data without hardware support, so we have to optimize the hardware. In the early stages of the application, often need to adjust the hardware optimization, many performance bottlenecks and get very good solve, but as the database scale growing, we need to turn the optimization to focus on comparative economic soft optimization level [21-22].

\subsection{The Security Enhancement of the Oracle Database}

Because people tend to use the database system management of the various data structure applications, database security problem is very common, in dealing with the security issues, people or solution focused on database system, or the attribute protective mechanism in the application code, these are misconceptions. In fact, database system cannot solve the problem of all the database security application system must also be simplified with the help of a database system security and security solutions.

According to the literature review, the primary threat for Oracle database could be listed as the following aspects. (1) Elevated privileges. For Oracle database, elevated privileges refer to low permissions users use opportunely, theft, or illegal way to get a higher authority, even the database administrator permissions. (2) Try to log in. For Oracle database is trying to $\log$ in with some sort of automated processes, through all alphanumeric combinations to try to crack a user name and password. (3) The influence of computer operating system commands to the database security. As the database of external barrier of the normal work of operating system security directly affect the security of database. (4) SQL injection attacks. Using SQL injection multistage attacks can provide interactive access to the operating system. Especially for large database security threat users through the SQL command inserted into a Web form submission or 
enter the domain name or page requests a query string, eventually to cheat the server performs malicious command, some users want to know the data [23-26].

Oracle provides a user resource files to manage the user password, restrict users to use the function of the system and database resources, below, for example, to create a resource file.

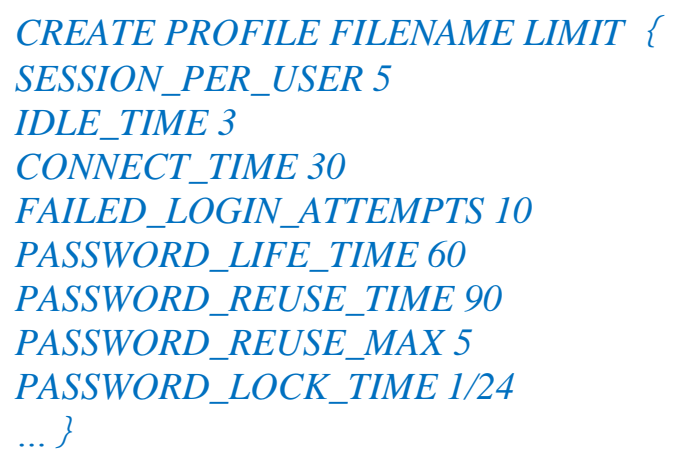

General audit functions and database to track implementation of SQL statements, but in the audit record does not contain the affected data values. If you need to track the actual affected data, it shall be used based on the value of the audit function used in Oracle database trigger to achieve this purpose which will largely enhance the robustness. In order to ensure the data in the database security and stability, to logical backup database in real time, physical backup, offline backup, online backup, even if a computer malfunctions, such as the medium damage, abnormal software system, and so on and so forth, we can through the backup recovery of the different level to make the Oracle database system returned to normal.

\section{The Enterprise Resource Management System and Evaluation Model}

\subsection{The Auxiliary Performance Evaluation Mechanism}

In theory, involves testing the validity of theory. Because, entrepreneurial performance as the most important of dependent variable, or the ultimate dependent variable, it is the standard for testing various interpretation of entrepreneurship theory and foresight. If entrepreneurship performance structure is not clear, the explanatory power of entrepreneurship theory and the predictive power won't get inspection effectively, the relative merits of several competing theories will not be able to judge, it will hinder development of theory of entrepreneurship. In practice, the role of performance indicators with a baton that kind of performance indicators, means something important, people will efforts in the direction of the indicators to measure and not measure what people will ignore anything. Unclear of entrepreneurship performance structure, it will be hard to judge what kind of management method is more effective in the practice of entrepreneurship and also cannot effectively guide the business practices.

Of the interaction between the elements is to point to: a factor variable, not only can cause change is decided by its system of fitness value, can also because other elements associated with the decision system of fitness value change. System overall fitness values change depends on the change of the two parts as the formula 4 defines the process [2731].

$$
f_{\text {evaluation }}=\frac{1}{N} \sum_{i=1}^{N} f_{i}\left(d_{1}, d_{i 1}, \cdots d_{i k}\right)
$$

The knowledge structure of information system concept modeling are certain domain modelers themselves based on the theory of the cognitive modeling method using specific representation theory domain process, in this process, modeler is the main body, the 
theory of domain is an object, the modeling method is a means as says is the result of theory field.

Concept modeling method of a variety of it is from the cognitive mode and the diversity of the said method, and the diversity of the two is the result of the complexity of the objective world and subjective world. Therefore, variety of modeling method is inevitable phenomenon, the key is how to evaluate its performance, how to choose to use. In the following formulas, we demonstrate the interactive modelling procedure.

$$
\begin{gathered}
\dot{q}^{(I)}=N^{(I)}\left(q^{(I)}\right)+K^{(I)}\left(q^{(I)}, q^{(V)}\right) \\
\dot{q}^{(V)}=N^{(V)}\left(q^{(V)}\right)+K^{(V)}\left(q^{(I)}, q^{(V)}\right)
\end{gathered}
$$

Based on the theory of the cooperation, the collaborative system control parameters include two categories, external and internal parameters external parameter refers to the system the impact of the external environment factors on the system of internal forces for venture capital and intellectual capital, is the most important factors of external absence made the investment environment and related policies, because practice shows that economic policy and laws and regulations constraint will greatly influence the venture capital and intellectual capital for the promotion of the enterprise performance. Because of the risk capital injection, enterprise to provide the capital value-added services to businesses can make enterprise intellectual capital level change and the change will make the whole system from the original steady state to a stable state which is shown as the follows.

$$
\dot{q}_{1}=r_{1} q_{1}+\alpha q_{1} q_{2}
$$

Because of the usefulness of a conceptual model only considered the modeling method can realize the extent of the expected goal and did not consider in order to achieve this goal, the price of the modeling method of ease of use only consider the ease of use modeling method and investigate its usefulness, so they can synthetically the performance concept modeling method of performance, and the modeling method of efficiency index is put forward to solve this problem. Concept modeling method of efficiency refers to the modeler in the modeling method is used to accomplish specific tasks in the whole process of resources for the benefit of the average consumption unit size which will be taken into consideration.

\subsection{The Enterprise Resource Management System Principles and Construction}

At present, there is no attach great importance to business process of chain management process control. Business process restructuring is associated with supply chain management subsystem on the specific stage of development or the implementation. In this phase must be aware of the business process control is very important, too much control links of supply or redundancy will seriously reduce the supply chain management process efficiency. Business control missing links, of course can also lead to supply chain business is out of control.

The current challenges of the ERP system could be summarized as the follows. (1) Trade in a dynamic environment, enterprise request vendor management inventory and warehouse direct delivery mode, the need to implement the production process of enterprise's response in time. Therefore, the supply and the demand, the labor market as well as each change on the machine performance demands of system thinking and response quickly, it's clearly a ERP is unable to solve at present. Therefore, often appear enterprise decision-makers must depend on the current data to make decisions. However, this kind of analysis is not complete, make the factory to the customer request delivery date unrealistic promises, as a result, the commitment to promise, make customers feel dissatisfied. (2) ERP market demand forecast as the external input, but the market sales prediction accuracy is not high, often in sales management is also difficult to solve. In this case to make the production plan often leads to a large amount of the shortage or excess 
supply products. Usually ERP software packages allow enterprises from the perspective of organization and inventory associate multiple distributions of the plant and equipment. However, even if several factories produce the same product ERP can't the factory as a unit can be substituted. To optimize the processes, we use corresponding structure design pattern to deal with the issues. We optimize the traditional models into the following parts. (1) Management subsystem on the basis of the strategic planning to strategic management subsystem output, output total cost management plan, sales plan as the technical development plan. (2) Strategic management subsystem directly to the business information management subsystem, the output is strategic planning. (3) Production management subsystem in the management subsystem and output of total cost management plan, sales plan, under control of technology development plan, output production plan and purchasing plan [32-33].

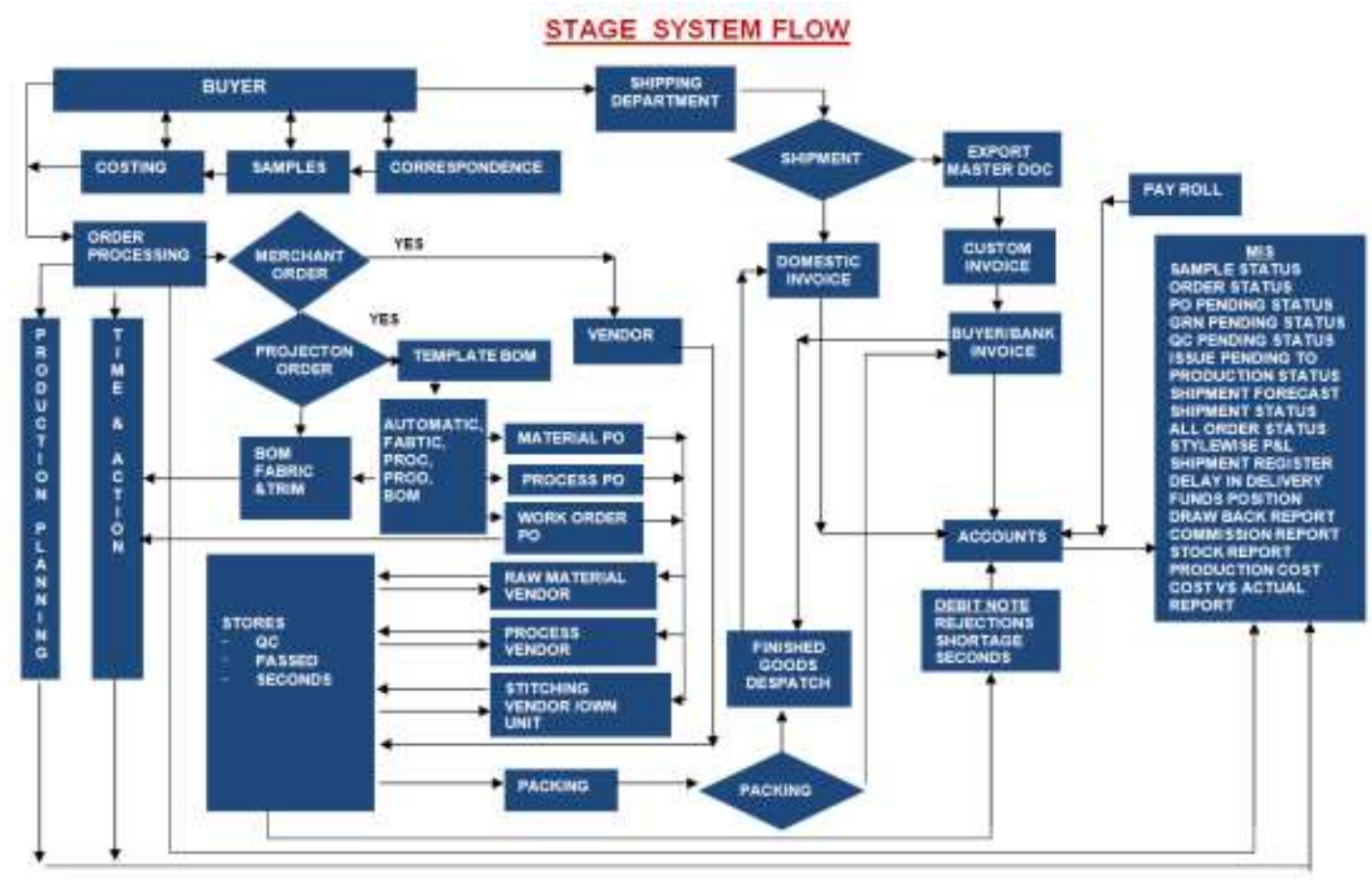

Figure 5. The Revised Flowchart of the ERP System

As illustrated in the figure 5, we show the revised flowchart of the ERP system. In process of development of extended ERP system is a very important aspect is the application of the use of Web database access technology. The general fusion of Web technology and database technology is a common concerned focus of the current database and the Web. Based on the model of software development is to model driven software development, namely, in order to achieve the goal of system, the system is divided into several levels, at every level of related objects modeling and to establish the mapping relationship between each layer model, using artificial or the computer software to complete the information according to the mapping rules from one level to another level, the transformation between the winning goal results. In this case, due to the mapping relationship between the model and the model is described in the form of metadata, so when the mapping relationship between model change and do not need to modify the program code, we only need to modify the metadata. 


\section{Experiment and Verification}

In this part, we simulate the proposed method. The figure 6 shows the user interface of the proposed enterprise resource management system and the figure 7 demonstrate comparison experiment on the database robustness and efficiency.

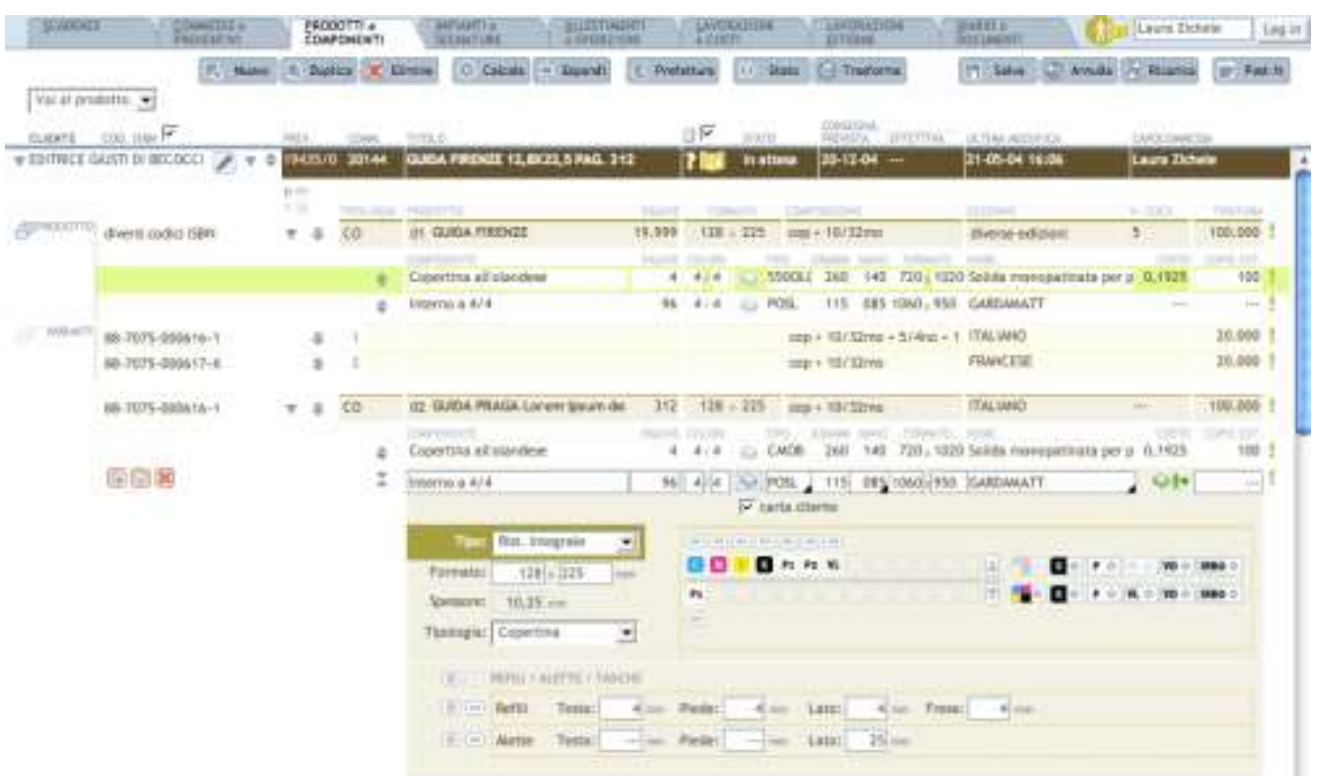

Figure 6. The Unser Interface Illustration of the Proposed Enterprise Resource Management System
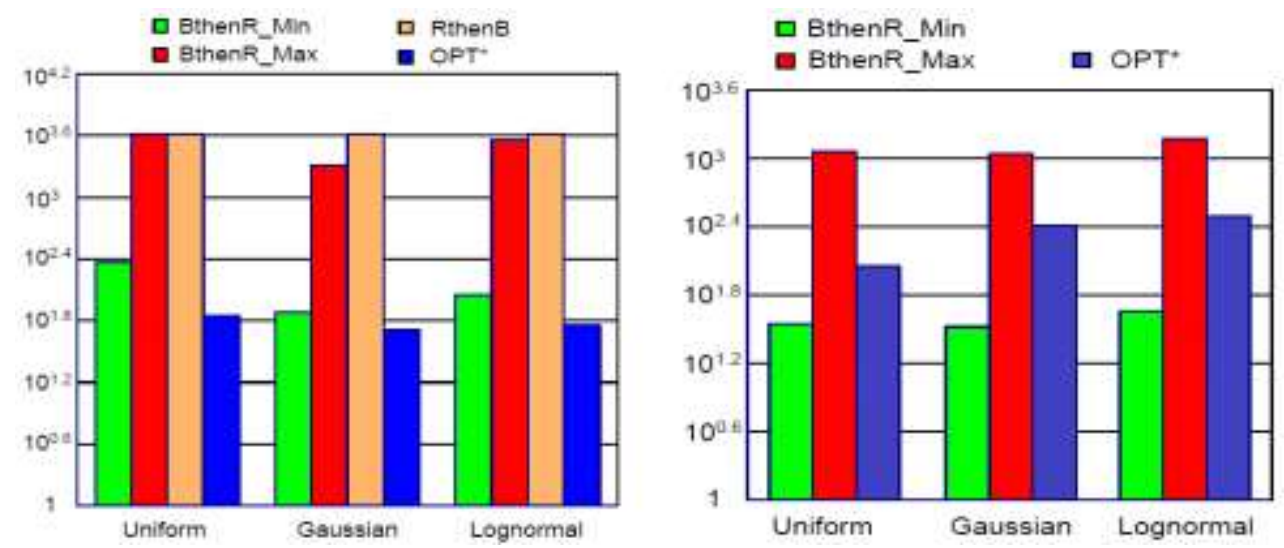

\section{Figure 7. The Comparison Experiment on the Database Robustness and Efficiency}

If enterprise performance of the supply of products or products has further demand, it can put forward to suppliers. Supplier should be according to the situation to improve their own production or the service, as well as a feedback to the enterprise users. These can be done by publishing information. In order to make the system can be suitable for smallest and the medium-sized enterprises, considering release information on the Internet, wants be particular about, real-time information to constantly update, every enterprise product information and forms, various parameters is not the same, so, in the design of generating product information page, using the dynamic generation technology better solve the problem. Our user interface combines the state-of-the-art design pattern to make it more feasible. The robustness and efficiency test shows that our method out performs compared with the other approaches. 


\section{Conclusion and Summary}

In this paper, we analyze the enterprise resource management system and related auxiliary performance evaluation mechanism based on Oracle database optimization and the security enhancement. Oracle is a great big relational database, has been widely used in the practical application. A selection of the size of the Oracle for database application system is compared commonly big, number of users is more, so efficiency is very important for such a system. We need to in the process of structure system and the system is running based on the actual situation to optimize application system to adjust at any time and make the application system more efficient. How to use the limited resources of a computer system to serve more users, how to ensure user's response speed and quality of service, all these problems belong to the category of database operation efficiency optimization. Our research combines the modern structure optimization techniques to optimize the database system and apply it into modern enterprise resource management system with the new designed user interface. The experiment result proves the robustness and efficiency of the system. According to the simulation result, the overall efficiency is enhanced $35 \%$ compared with the other approaches.

\section{References}

[1] G. McKeown, M. Valstar, R. Cowie, M. Pantic and M. Schröder, "The semaine database: Annotated multimodal records of emotionally colored conversations between a person and a limited agent", Affective Computing, IEEE Transactions on, vol. 3, no. 1, (2012), 5-17.

[2] E. Bardissi, W. Andrew, S. F. Aranki, S. Sheng, S. M. O’Brien, C. C. Greenberg and J. S. Gammie, "Trends in isolated coronary artery bypass grafting: an analysis of the Society of Thoracic Surgeons adult cardiac surgery database", The Journal of Thoracic and Cardiovascular Surgery, vol. 143, no. 2 (2012), pp. 273-281.

[3] K. Smolina, F. L. Wright, M. Rayner and M. J. Goldacre, "Determinants of the decline in mortality from acute myocardial infarction in England between 2002 and 2010: linked national database study", Bmj, vol. 344, (2012), d8059.

[4] V. Wakelam, E. Herbst, J. C. Loison, I. W. M. Smith, V. Chandrasekaran, B. Pavone and N. G. Adams, "A kinetic database for astrochemistry (KIDA)", The Astrophysical Journal Supplement Series, vol. 199, no. 1, (2012), p. 21.

[5] J. Rezác, K. E. Riley and P. Hobza, "S66: A well-balanced database of benchmark interaction energies relevant to biomolecular structures", Journal of chemical theory and computation, vol. 7, no. 8, (2011), pp. 2427-2438.

[6] K. Jimmy, T. A. Jahan and M. R. Hoopmann, "Comet: An open- source MS/MS sequence database search tool", Proteomics, vol. 13, no. 1, (2013), pp. 22-24.

[7] Y. Liu, A. Wirawan and B. Schmidt, "CUDASW++ 3.0: accelerating Smith-Waterman protein database search by coupling CPU and GPU SIMD instructions", BMC bioinformatics, vol. 14, no. 1, (2013), p. 117.

[8] S. I. F. G. N. Nes and S. M. S. M. M. Kersten, "MonetDB: Two decades of research in column-oriented database architectures", Data Engineering, (2012), p. 40.

[9] Z. Xu, Y. C. Tu and X. Wang, "PET: reducing database energy cost via query optimization", Proceedings of the VLDB Endowment, vol. 5, no. 12, (2012), pp. 1954-1957.

[10] K. Ramachandra, R. Guravannavar and S. Sudarshan, "Program analysis and transformation for holistic optimization of database applications", In Proceedings of the ACM SIGPLAN International Workshop on State of the Art in Java Program analysis, (2012), pp. 39-44.

[11] M. G. Cole and N. Dendukuri, "Risk factors for depression among elderly community subjects: a systematic review and meta-analysis", American Journal of Psychiatry, (2014).

[12] N. M. Batalha, J. F. Rowe, S. T. Bryson, T. Barclay, C. J. Burke, D. A. Caldwell and J. L. Christiansen, "Planetary candidates observed by Kepler. III. Analysis of the first 16 months of data", The Astrophysical Journal Supplement Series, vol. 204, no. 2, (2013), p. 24.

[13] A. J. Vickers, A. M. Cronin, A. C. Maschino, G. Lewith, H. MacPherson, N. E. Foster, K. J. Sherman, C. M. Witt, K. Linde and Acupuncture Trialists' Collaboration, "Acupuncture for chronic pain: individual patient data meta-analysis", Archives of internal medicine, vol. 172, no. 19, (2012), pp. 1444-1453.

[14] N. O'Rourke, R. Psych and L. Hatcher, "A step-by-step approach to using SAS for factor analysis and structural equation modeling", Sas Institute, (2013).

[15] W. W. Cooper, L. M. Seiford and J. Zhu, "Handbook on data envelopment analysis", Springer Science \& Business Media, vol. 164, (2011). 
[16] J. Wang, H. Wang, Y. Zhou and N. McDonald, "Multiple kernel multivariate performance learning using cutting plane algorithm", arXiv preprint arXiv:1508.06264, (2015).

[17] P. Khatri, S. D. Yeatts, M. Mazighi, J. P. Broderick, D. S. Liebeskind, A. M. Demchuk and P. Amarenco, "Time to angiographic reperfusion and clinical outcome after acute ischaemic stroke: an analysis of data from the Interventional Management of Stroke (IMS III) phase 3 trial”, The Lancet Neurology, vol. 13, no. 6 , (2014), pp. 567-574.

[18] R. Honea, T. J. Crow, D. Passingham and C. E. Mackay, "Regional deficits in brain volume in schizophrenia: a meta-analysis of voxel-based morphometry studies", American Journal of Psychiatry, (2014).

[19] P. Blanchard, "Taxane-cisplatin-fluorouracil as induction chemotherapy in locally advanced head and neck cancers: an individual patient data meta-analysis of the meta-analysis of chemotherapy in head and neck cancer group", Journal of Clinical Oncology, vol. 31, no. 23, (2013).

[20] R. Coppi, P. D’Urso and P. Giordani, "Fuzzy and possibilistic clustering for fuzzy data”, Computational Statistics \& Data Analysis, vol. 56, no. 4, (2012), pp. 915-927.

[21] D. Jenter and F. Kanaan, "CEO turnover and relative performance evaluation", The Journal of Finance, (2015).

[22] R. Bakhshi, L. Cloth, W. Fokkink and B. R. Haverkort, "Mean-field framework for performance evaluation of push-pull gossip protocols", Performance Evaluation, vol. 68, no. 2, (2011), pp. 157-179.

[23] P. T. Lauzier, J. Tang and G. H. Chen, "Prior image constrained compressed sensing: Implementation and performance evaluation", Medical physics, vol. 39, no. 1, (2012), pp. 66-80.

[24] S. Ooi, S. Morimoto, M. Sanada and Y. Inoue, "Performance evaluation of a high-power-density PMASynRM with ferrite magnets", Industry Applications, IEEE Transactions on, vol. 49, no. 3, (2013), pp. 1308-1315.

[25] S. Chen, "Performance evaluation of radar and satellite rainfalls for Typhoon Morakot over Taiwan: Are remote-sensing products ready for gauge denial scenario of extreme events?", Journal of Hydrology, vol. 506, (2013), pp. 4-13.

[26] R. Lin, X. Zhao and G. Wei, "Models for selecting an ERP system with hesitant fuzzy linguistic information", Journal of Intelligent \& Fuzzy Systems: Applications in Engineering and Technology, vol. 26, no. 5, (2014), pp. 2155-2165.

[27] P. Ifinedo, "Examining the influences of external expertise and in-house computer/IT knowledge on ERP system success", Journal of Systems and Software, vol. 84, no. 12, (2011), pp. 2065-2078.

[28] Y. Zeng and M. J. Skibniewski, "Risk assessment for enterprise resource planning (ERP) system implementations: a fault tree analysis approach", Enterprise Information Systems, vol. 7, no. 3, (2013), pp. 332-353.

[29] J. Wei and Y. S. Ma, "Design of a feature-based order acceptance and scheduling module in an ERP system", Computers in Industry, vol. 65, no. 1, (2014), pp. 64-78.

[30] C. H. Jin, Y. J. Kwon, J. Cui, S. H. Lee and S. Y. Kim, "A Study of ERP Construction Strategies by System Factors: Based on Case Studies of Korea and China Firms", Journal of the Korea Industrial Information System Society, vol. 17, no. 3, (2012), pp. 69-81.

[31] F. Chen, "The study on ERP system evaluation based on fuzzy analytic hierarchy process method", International Journal of Digital Content Technology and its Applications, vol. 6, no. 22, (2012), p. 231.

[32] H. Lv, and L. L. Dong, "Research on the Evaluation Index System of ERP Implementation Performance Based on the Fuzzy Algorithm”, In Advanced Materials Research, vol. 717, (2013), pp. 904-908.

[33] P. Upadhyay, S. Jahanyan and P. K. Dan, "Factors influencing ERP implementation in Indian manufacturing organisations: A study of micro, small and medium-scale enterprises", Journal of Enterprise Information Management, vol. 24, no. 2, (2011), pp. 130-145. 
International Journal of Future Generation Communication and Networking Vol. 10, No. 1 (2017) 